\title{
SUSTAINABILITY OF WHEAT (Triticum aestivum L.) YIELD AND IMPROVEMENT OF SEED QUALITY THROUGH PROBIOTIC AND ORGANIC SOIL AMENDMENT
}

\author{
M.A. Hossain ${ }^{1,2}$, S.M.S. Islam ${ }^{1}$ and M.M. Hasan ${ }^{3}$ \\ ${ }^{1}$ Plant Biotech.\& Genetic Eng. Lab., Inst. of Biol. Sci., University of Rajshahi \\ ${ }^{2}$ Directorate of Secondary and Higher Education, Dhaka-1000 \\ 3Plant Path. Lab., Agron. \& Agril. Extn. Dept., University of Rajshahi \\ Corresponding E-mail:mahmudul742001@yahoo.com
}

(Received: 27 November 2020, Accepted: 31 January 2021)

Keywords: Poultry manure, vermicompost, green manure, yield, seed quality, wheat

\begin{abstract}
In search of alternative counter to harmful effects of chemical fertilizers on soils and environment, probiotic and organic manures-based fertilizer management options need to be evaluated. The experiments were designed as randomized complete block design (RCBD) consisting of three wheat varieties and nine soil amendment treatments. The result revealed that organic amendments had prominent and variable effects on studied parameters and statistically at par with chemical fertilizer. Some yield-associated parameters like spike length, spikeletsspike ${ }^{-1}$, fertile spikelets spike $e^{-1}$, grains spike $e^{-1}$, grains weight spike ${ }^{-1}$ and 1000 -grain weight were significantly influenced by organic amendments. Moreover, grain yield and straw yield were increased $73 \%$ and $27 \%$, respectively under the treatment of poultry manure combination in comparison with control. In addition, the seed quality characters viz. germination, vigor index and total soluble protein content also exhibited significant improvement showing 23\%, 44\%and 17\%, respectively by poultry manure + vermicompost + green manure. The above findings showed that to apply poultry manure + vermicompost + green manure as an effective soil amendment option and to obtain good yield and quality seed of wheat.
\end{abstract}

\section{Introduction}

In Bangladesh, wheat occupies 4\% of the total cropped area and 11\% of the cropped area in the Rabi season and contributes $7 \%$ to the total output of food cereals (BBS, 2008). In Bangladesh, the average yield of wheat is $3.16 \mathrm{t} \mathrm{ha}^{-1}$ (BBS, 2017), which is below the achievable yield of $4.5 \mathrm{t} \mathrm{ha}^{-1}$ (BARI, 2011). Alam et al. (2013) stated two reasons for the yield gap as (i) biotic factors including poor quality seeds and seedlings, insects, diseases, weeds and rodents; and (ii) abiotic factors including soil, nutrients and water. However, many reasons for this yield gap remain unexplained. Nutrients availability of soils is declining with time pace but which had been rich in the past. Among the different agricultural inputs, fertilizer is the most important one and nearly 50\% of the modern agricultural production depends on this insert (Pradhan, 1992). The non-judicial and imbalanced use of chemical fertilizer generate hazards on soil, environment and human health which led the growers' considerable attention to turn organic manures application for sustainable production practices (Singh et al., 2018). It is authentic that fairly good soil fertility and plant nutrients are important to farming, whether the practices are considered "conventional" or "sustainable" (Hue and Silva, 2000). Yadvinder-Singh 
et al. (2008) stated organic materials like crop residues, green manure, and animal manure show great influence on soil productivity. Therefore, probiotic and organic manures application should be evaluated on agricultural land reclamation and crop yield potentiality aspects in Bangladesh. In this view, the investigation was carried out to assess the effect of probiotic and organic manure amendment soil on the yield and seed quality of wheat.

\section{Materials and Methods}

The present piece of research work was conducted in the Institute of Biological Sciences (IBS) and Plant Pathology Laboratory, Department of Agronomy and Agricultural Extension, University of Rajshahi, Bangladesh during the period from July 2016 to June 2019. The experimental field is geographically situated at $24^{\circ} 17^{\prime \prime} \mathrm{N}$ latitude and $88^{\circ} 28^{\prime \prime} \mathrm{E}$ longitude at an elevation of $20 \mathrm{~m}$ above the sea level belonging to the Agro-ecological Zone-11(AEZ-11). The soil of the experimental field is characterized by poorly drained with moderate permeable, loamy and slightly alkaline $(\mathrm{pH}=8.10)$ in nature. Before 2016, the field was occupied with barley production for two years. The experiment was laid out in randomized complete block design (RCBD) with three replications. Three wheat varieties viz. BARI Gom-28 $\left(\mathrm{V}_{1}\right)$, BARI Gom-29 $\left(\mathrm{V}_{2}\right)$, BARI Gom-30 $\left(\mathrm{V}_{3}\right)$ and nine soil amendment treatments viz. control $\left(\mathrm{T}_{0}\right)$, rice straw + vermicompost + green manure $\left(\mathrm{T}_{1}\right)$, cow dung + vermicompost + green manure $\left(\mathrm{T}_{2}\right)$, compost + vermicompost + green manure $\left(T_{3}\right)$, poultry manure + vermicompost + green manure $\left(T_{4}\right)$, Trichoderma harzianum + vermicompost + green manure $\left(\mathrm{T}_{5}\right)$, mungbean residue + vermicompost + green manure $\left(T_{6}\right)$, Trichoderma viride + vermicompost + green manure $\left(T_{7}\right)$ and chemical fertilizer $\left(\mathrm{T}_{8}\right)$ were used. The unit plot was $5.0 \mathrm{~m}^{2}$ having the plot to plot $0.5 \mathrm{~m}$, bed to bed $0.25 \mathrm{~cm}$ distances and $1 \mathrm{~m}$ from surrounding the boundary. Seeds of Dhaincha (Sesbania rostrata) were sown at the rate of $50 \mathrm{~kg} \mathrm{ha}^{-1}$ to the respective plots and after 50 days of sowing young succulent green plants were incorporated into the soil. Crop residues (rice straw, mungbean residue), cow dung, compost and poultry litter were applied @ $10 \mathrm{t} \mathrm{ha}^{-1}$ before 7 days of sowing. Vermicompost was applied @ $5 \mathrm{t} \mathrm{ha}{ }^{-1}$ and Trichoderma spp. suspension (1 $10^{6} \mathrm{cfu} \mathrm{g}^{-1} @ 5 \mathrm{~kg} \mathrm{ha}^{-1}$ ) before sowing. In the case of chemical treatment, one-third of urea (200 kg ha-1), TSP $160 \mathrm{~kg} \mathrm{ha}^{-1}$, MoP $45 \mathrm{~kg} \mathrm{ha}^{-1}$ and Gypsum $115 \mathrm{~kg} \mathrm{ha}^{-1}$ were used as basal dose (BARI, 2014). The rest of the urea was applied as two installments one at 21 DAS and another at 55 DAS.

\section{Data recording}

In the case of yield and yield attributes, ten spikes were selected randomly from each experimental plot for recording data on spike length, spikeletsspike ${ }^{-1}$, fertile spikelets spike ${ }^{-1}$, grains spike $e^{-1}$ and grain weight spike $e^{-1}$, while 1000 -grain weight, grain yield and straw yield of crops were recorded which were collected from one square meter area the center of each unit plot.

\section{Harvest index (\%)}

The harvest index denotes the ratio of grain yield (economic yield) to biological yield and was calculated with the following formula:

$$
\text { Harvest index }(\%)=\frac{\text { Grain yield }}{\text { Biological yield }} \times 100
$$

Here, Biological yield $=$ Grain yield + straw yield . 


\section{Determination of seed germination}

Four hundred seeds of each treatment were taken for germination test as recommended by ISTA (1985). Germination of seeds was collected regularly for up to 10 days. Percentage of germination was determined as follows:

$$
\text { Germination }(\%)=\frac{\text { No. of germinated seedling }}{\text { No. of seed set for germination }} \times 100
$$

\section{Vigor index}

After setting seeds, the germination percentage was calculated from the final count. Vigor index was found out by using the following formula (Maguire, 1962):

$$
\begin{aligned}
\text { Vigor index }= & \frac{\text { No. of normal seedlings (First count) }}{\text { Days to first count }}+------+ \\
& \frac{\text { No. of normal seedlings (Final count) }}{\text { Days to final count }}
\end{aligned}
$$

\section{The total soluble protein content of seed}

A technique formerly defined by Guy et al. (1992) was performed with some modification to estimate the total soluble protein of seed using a spectrophotometer.

\section{Statistical analysis}

The recorded data were compiled and tabulated for statistical analysis. The trial data for all parameters were analyzed statistically for analysis of variance (ANOVA) throughr-studio (http://www.rstudio.com/) of "Agricolae" package (https://CRAN.R project.org/package=agricolae) and Duncan's multiple range test (DMRT) was performed for comparing treatment means(Gomez and Gomez, 1984).

\section{Results and Discussion}

\section{Effect of soil amendment on yield and yield attributes}

\section{Spike length}

The spike length was affected significantly by various soil amendment treatments (Table 1). In the final year, it has been found that the treatment $T_{8}$ (chemical fertilizer) produced the maximum spike length $(8.60 \mathrm{~cm})$ which was followed by $\mathrm{T}_{4}$ (poultry manure + vermicompost + green manure) $(8.43 \mathrm{~cm})$ while the smallest spike length recorded from $T_{0}(7.00 \mathrm{~cm})$ It might be due to higher nutrient concentration and moisture retention of the soil amended with poultry manure combination treatment that led to the formation of longer spike length. This result was correlated with Ahmed et al. (2002). Nevertheless, green manure fortified poultry manure keeps the $\mathrm{N}$ balance which may enhanced the length of spike of wheat. The above statement was also supported by Nguyen et al. (1995).

\section{Spikelets spike ${ }^{-1}$ and fertile spikelets spike $e^{-1}$}

The spikelets spike $e^{-1}$ is treated as an important element of wheat grain yield (Sabaghina et al., 2014). From Table 1, it revealed that spikelets spike $e^{-1}$ varied significantly from 13.80 to 17.97 where the maximum and the minimum value for this parameter were recorded from $T_{8}$ and $T_{0}$. 
Nitrogen-rich organic manures may have a positive effect on the growth and yield attributes of wheat where higher fertile spikelet spike ${ }^{-1}$ from $T_{8}$ (17.24) and $T_{4}(17.00)$ but 13.22 was the lowest obtained from $\mathrm{T}_{0}$. These findings were partially correlated with the observation of Siavoshi et al. (2011).

\section{Grains spike $e^{-1}$}

Grains spike $e_{\text {is }}$ significantly influenced by soil amendments. During the research period, a significantly higher number of grains spike ${ }^{-1}$ was recorded from the treated plot in comparison with the control plot. The rank of treatments were $T 8>T_{4}>T_{5}>T_{7}>T_{3}>T_{6}>T_{2}>T_{1}>T_{0}$ (Table 2). The maximum number of grains spike ${ }^{-1}$ (45.73) was recorded from $T_{8}$ which was statistically similar to $\mathrm{T}_{4}(44.80)$ and the least value from $\mathrm{T}_{0}$ (33.67). Considering the organic soil amendment treatments, poultry manure $\left(\mathrm{T}_{4}\right)$ performed the better response; such result might be due greater amount of $\mathrm{N}$ within it, which produced more dry matter and partitioning it towards grains which enhanced the grains spike ${ }^{-1}$. This result was confirmatory with the outcomes of Shah et al. (2010) and Mukhtiar et al. (2018).

Table 1. Effect of organic and probiotic soil amendments on yield and yield contributing characters of wheat

\begin{tabular}{ccccccccccc}
\hline Treatments & \multicolumn{3}{c}{$\begin{array}{c}\text { Spike length } \\
\text { (cm) }\end{array}$} & \multicolumn{3}{c}{$\begin{array}{c}\text { Spikeletsspike } \\
\text { (no.) }\end{array}$} & \multicolumn{3}{c}{ Fertile spikeletsspike } \\
(no.)
\end{tabular}

In the column, mean values bearing similar letter(s) or without letter are similar and those having dissimilar letters are differed significantly as per Duncan's Multiple Range Test. $T_{0}=$ control, $T_{1}=$ rice straw + vermicompost + green manure, $T_{2}=$ cow dung + vermicompost + green manure, $T_{3}=$ compost + vermicompost + green manure, $\mathrm{T}_{4}=$ poultry manure + vermicompost + green manure, $\mathrm{T}_{5}=T$. harzianum + vermicompost + green manure, $\mathrm{T}_{6}=$ mungbean residues + vermicompost + green manure, $\mathrm{T}_{7}=T$. viride + vermicompost + green manure, $\mathrm{T}_{8}=$ chemical fertilizer, $\mathrm{LS}=$ Level of significance, ${ }^{* *}=1 \%$ level of significance, $C V=$ Coefficient of variation 


\section{Grains weight spike ${ }^{-1}$}

As a yield component, grains weight spike ${ }^{-1}$ of wheat is an important factor that contributes to the grain yield (Peltonen-Sainio et al., 2007). Grain weight spike $e^{-1}$ at harvest varied significantly due to different soil amendment treatments (Table 2). Among the different soil amendment treatments, $\mathrm{T}_{4}(2.14 \mathrm{~g})$ performed better than other treatments. The rest of the treatments were also showed as descending by $\mathrm{T}_{5}(2.06 \mathrm{~g}), \mathrm{T}_{7}(2.01 \mathrm{~g}), \mathrm{T}_{3}(1.99 \mathrm{~g}), \mathrm{T}_{2}(1.93 \mathrm{~g}), \mathrm{T}_{6}(1.89 \mathrm{~g}), \mathrm{T}_{1}$ $(1.86 \mathrm{~g})$, while the lowest one in $\mathrm{T}_{0}(1.60 \mathrm{~g})$. The advancement of grain weight spike $e^{-1}$ that provided with poultry manure treatment combination might be due to balanced nutrient stock during the grain filling stage and improvement of soil fertility. A similar result was also reported by Garg and Bahla (2008).

\section{0-grain weight}

For cereal crops, 1000-grain weight is an important factor to determine the yield and yield attributes (Metho et al., 1998). The data of 1000-grain weight of wheat indicated that chemical fertilizer $\left(T_{8}\right)$ and poultry manure combination $\left(T_{4}\right)$ significantly increased the weight $(54.12 \mathrm{~g}$ and $53.90 \mathrm{~g}$ ) while plants that did not receive any soil amendments $\left(\mathrm{T}_{0}\right)$ showed the lowest $(48.78 \mathrm{~g})$ might be due to availability of nutrients throughout the growing period and improvement of soil characters. This outcome was agreed with Ahmed et al. (2017).

Table 2. Effect of organic and probiotic soil amendments on yield and yield contributing characters of wheat

\begin{tabular}{|c|c|c|c|c|c|c|c|c|c|}
\hline \multirow[b]{2}{*}{ Treatments } & \multicolumn{3}{|c|}{ Grains spike $^{-1}$ (no.) } & \multicolumn{3}{|c|}{${\text { Grain weight spike }{ }^{-1}(\mathrm{~g})}$} & \multicolumn{3}{|c|}{ 1000-grainweight (g) } \\
\hline & $\begin{array}{l}2016- \\
2017\end{array}$ & $\begin{array}{c}2017- \\
2018\end{array}$ & $\begin{array}{c}2018- \\
2019\end{array}$ & $\begin{array}{l}2016- \\
2017\end{array}$ & $\begin{array}{c}2017- \\
2018\end{array}$ & $\begin{array}{c}2018- \\
2019\end{array}$ & $\begin{array}{l}2016- \\
2017\end{array}$ & $\begin{array}{c}2017- \\
2018\end{array}$ & $\begin{array}{c}2018- \\
2019\end{array}$ \\
\hline \multirow[b]{2}{*}{$\mathrm{T}_{0}$} & $36.88 f$ & $35.04 e$ & $33.67 \mathrm{i}$ & $1.66 \mathrm{~g}$ & $1.62 \mathrm{~g}$ & $1.60 \mathrm{~g}$ & $49.52 \mathrm{f}$ & $48.61 e$ & $48.78 \mathrm{i}$ \\
\hline & & & & \pm 0 & & \pm 0.04 & \pm 0.44 & & \\
\hline \multirow{2}{*}{$\mathrm{T}_{1}$} & $36.98 f$ & $38.42 \mathrm{~d}$ & $39.53 \mathrm{~h}$ & $1.74 \mathrm{f}$ & $1.80 f$ & $1.86 f$ & $50.03 f$ & 51. & $51.30 \mathrm{~h}$ \\
\hline & \pm 0.22 & \pm 0.23 & \pm 0.88 & \pm 0.02 & & \pm 0.04 & \pm 0.37 & & \\
\hline \multirow{2}{*}{$\mathrm{T}_{2}$} & $38.11 e$ & 39.36d & $40.67 f$ & $1.80 \mathrm{ef}$ & $1.88 \mathrm{de}$ & $1.93 e$ & 51.19de & 51. & $51.95 f$ \\
\hline & \pm 0.21 & \pm 0.26 & \pm 0.96 & \pm 0.03 & \pm 0.03 & \pm 0.05 & \pm 0.33 & & \pm 0.33 \\
\hline \multirow{2}{*}{$\mathrm{T}_{3}$} & $38.76 \mathrm{~d}$ & $40.51 c$ & $41.58 e$ & $1.83 \mathrm{de}$ & $1.91 \mathrm{~d}$ & $1.99 \mathrm{~d}$ & $51.77 \mathrm{~cd}$ & 52. & $52.49 e$ \\
\hline & \pm 0.21 & \pm 0.39 & \pm 1.02 & \pm 0.02 & \pm 0 & \pm 0.04 & \pm 0.19 & & \pm 0.34 \\
\hline \multirow{2}{*}{$\mathrm{T}_{4}$} & $42.11 b$ & $43.50 a$ & $44.80 \mathrm{~b}$ & $1.98 b$ & $2.10 \mathrm{~b}$ & $2.14 b$ & $52.94 \mathrm{ab}$ & 53.51ab & $53.90 b$ \\
\hline & \pm 0.40 & \pm 0.59 & \pm 1.13 & \pm 0.02 & \pm 0.02 & \pm 0.05 & \pm 0.27 & \pm 0 & \pm 0.39 \\
\hline \multirow{2}{*}{$\mathrm{T}_{5}$} & $40.17 \mathrm{c}$ & $41.59 b$ & $43.14 c$ & $1.93 \mathrm{bc}$ & $2.02 \mathrm{c}$ & $2.06 \mathrm{c}$ & $52.27 \mathrm{bc}$ & 52.98abc & $53.19 c$ \\
\hline & \pm 0.22 & \pm 0 & \pm 1.0 & \pm 0.03 & \pm 0.02 & \pm 0.05 & \pm 0.21 & \pm 0 & \pm 0.38 \\
\hline & $37.20 \mathrm{f}$ & $38.71 d$ & $40.23 \mathrm{~g}$ & 1.77 ef & $1.84 e f$ & $1.89 \mathrm{f}$ & $50.48 \mathrm{ef}$ & $51.39 \mathrm{~cd}$ & $51.60 \mathrm{~g}$ \\
\hline & \pm 0.20 & \pm 0.27 & \pm 0.97 & \pm 0.03 & & \pm 0.05 & \pm 0.40 & & \pm 0.34 \\
\hline \multirow{2}{*}{$\mathrm{T}_{7}$} & $39.73 c$ & $40.83 b c$ & $42.50 \mathrm{~d}$ & $1.89 \mathrm{~cd}$ & $1.99 \mathrm{c}$ & $2.01 d$ & $51.97 \mathrm{bcd}$ & $52.51 \mathrm{a}-\mathrm{d}$ & $52.64 d$ \\
\hline & 295 & & \pm 1.07 & \pm 0.02 & \pm 0.02 & \pm 0.05 & \pm 0.14 & & \pm 0.36 \\
\hline \multirow{2}{*}{$\mathrm{T}_{8}$} & $42.74 a$ & $44.41 \mathrm{a}$ & $45.73 a$ & $2.07 a$ & $2.15 a$ & $2.20 \mathrm{a}$ & $53.28 \mathrm{a}$ & $53.81 \mathrm{a}$ & $54.12 \mathrm{a}$ \\
\hline & \pm 0.37 & \pm 0.53 & \pm 1.04 & \pm 0.03 & \pm 0.03 & \pm 0.05 & \pm 0.30 & \pm 0.29 & \pm 0.40 \\
\hline LS & **** & *** & *** & $* *$ & $* *$ & $* *$ & *** & $* *$ & *** \\
\hline CV (\%) & 1.52 & 2.55 & 1.09 & 3.69 & 2.90 & 1.48 & 1.90 & 3.12 & 1.23 \\
\hline
\end{tabular}

In the column, mean values bearing similar letter(s) or without letter are similar and those having dissimilar letters are differed significantly as per Duncan's Multiple Range Test. $\mathrm{T}_{0}=$ control, $\mathrm{T}_{1}=$ rice straw + vermicompost + green manure, $T_{2}=$ cow dung + vermicompost + green manure, $T_{3}=$ compost + vermicompost + green manure, $\mathrm{T}_{4}=$ poultry manure + vermicompost + green manure, $\mathrm{T}_{5}=T$. harzianum + vermicompost + green manure, $\mathrm{T}_{6}=$ mungbean residues + vermicompost + green manure, $\mathrm{T}_{7}=T$. viride + vermicompost + green manure, $\mathrm{T}_{8}=$ chemical fertilizer, $\mathrm{LS}=$ Level of significance, ${ }^{* *}=1 \%$ level of significance, $\mathrm{CV}=$ Coefficient of variation 


\section{Grain yield}

Different yield component specifically spikesm ${ }^{-2}$ number and weight of grains spike ${ }^{-1}$ and 1000 grain weight mathematically functioned on yield of cereal crop (Thiry et al., 2002). Application of different soil amendments showed significant variation of grain yield of wheat (Figure 1,2 and 3 ). In the last year, influences of different treatments on grain yield were found to be ranked in that following descending order $\mathrm{T}_{8}\left(4.25 \mathrm{tha}^{-1}\right)>\mathrm{T}_{4}\left(4.09 \mathrm{tha} \mathrm{a}^{-1}\right)>\mathrm{T} 5\left(3.85 \mathrm{t} \mathrm{ha}^{-1}\right)>\mathrm{T}_{7}(3.64$ $\left.\mathrm{t} \mathrm{ha}^{-1}\right)>\mathrm{T}_{3}\left(3.60 \mathrm{t} \mathrm{ha}^{-1}\right)>\mathrm{T}_{2}\left(3.34 \mathrm{t} \mathrm{ha}^{-1}\right)>\mathrm{T}_{6}\left(3.27 \mathrm{t} \mathrm{ha}^{-1}\right)>\mathrm{T}_{1}\left(3.05 \mathrm{t} \mathrm{ha}^{-1}\right)>\mathrm{T}_{0}(2.36$ $\left.t \mathrm{ha}^{-1}\right)$. Better performance of $\mathrm{T}_{4}$ might be enhanced soil fertility and improvement of physical conditions, reduction of nutrient losses which collectively contributed to the higher grain yield. This result was in confirmed to that with the observation of Zhang et al. (2016).

\section{Straw yield}

Soil amendments significantly influenced the straw yield (Figure 1, 2 and 3). Among the different treatments, $\mathrm{T}_{8}\left(5.40 \mathrm{t} \mathrm{ha}^{-1}\right)$ performed superior. The rest of the treatments were also showed significant result ranked as descending by $\mathrm{T}_{4}\left(5.31 \mathrm{t} \mathrm{ha}^{-1}\right), \mathrm{T}_{5}\left(5.14 \mathrm{t} \mathrm{ha}^{-1}\right), \mathrm{T}_{7}\left(5.05 \mathrm{t} \mathrm{ha}^{-1}\right), \mathrm{T}_{3}$ (4.93 tha-1), $\mathrm{T}_{6}\left(4.83 \mathrm{t} \mathrm{ha}^{-1}\right), \mathrm{T}_{2}\left(4.73 \mathrm{tha}^{-1}\right)$ and $\mathrm{T}_{1}\left(4.60 \mathrm{t} \mathrm{ha}^{-1}\right)$, while the lowest one in $\mathrm{T}_{0}$ $\left(4.18 \mathrm{t} \mathrm{ha}^{-1}\right)$. This performance of organic manures might be due to the capability of abundant nutrients supply and the creation of a favorable growth environment. A similar result was also noted by Auti et al. (1999) and Kumar and Abraham (2018).

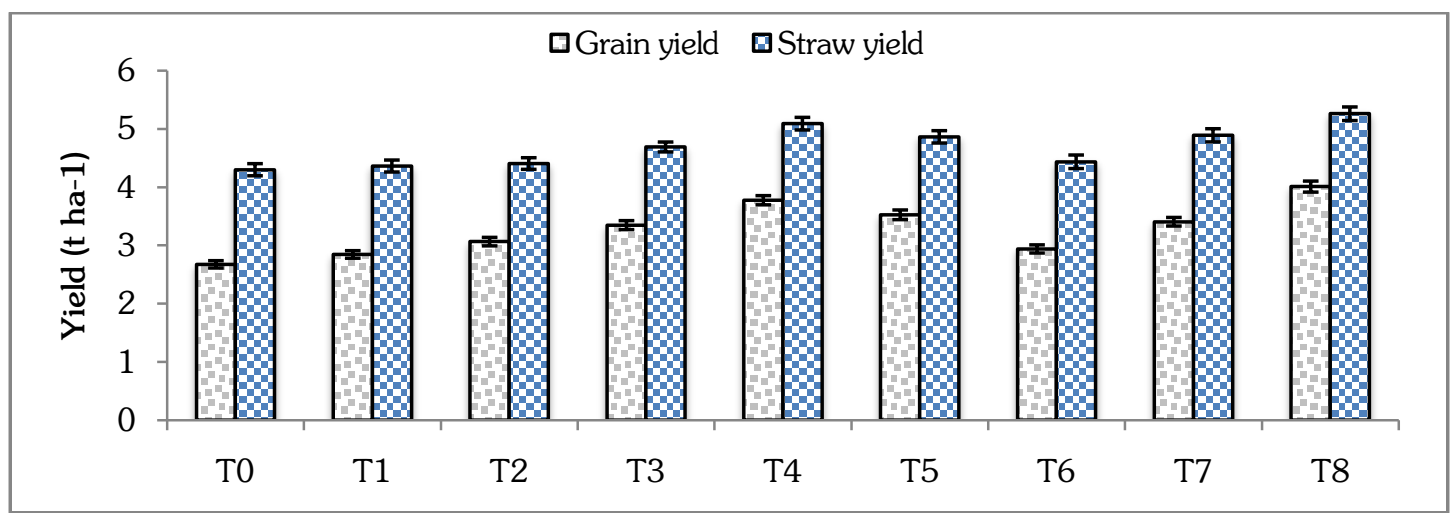

Fig. 1. Effect of organic and probiotic soil amendments on grain yield and straw yield of wheat in 2016-2017.

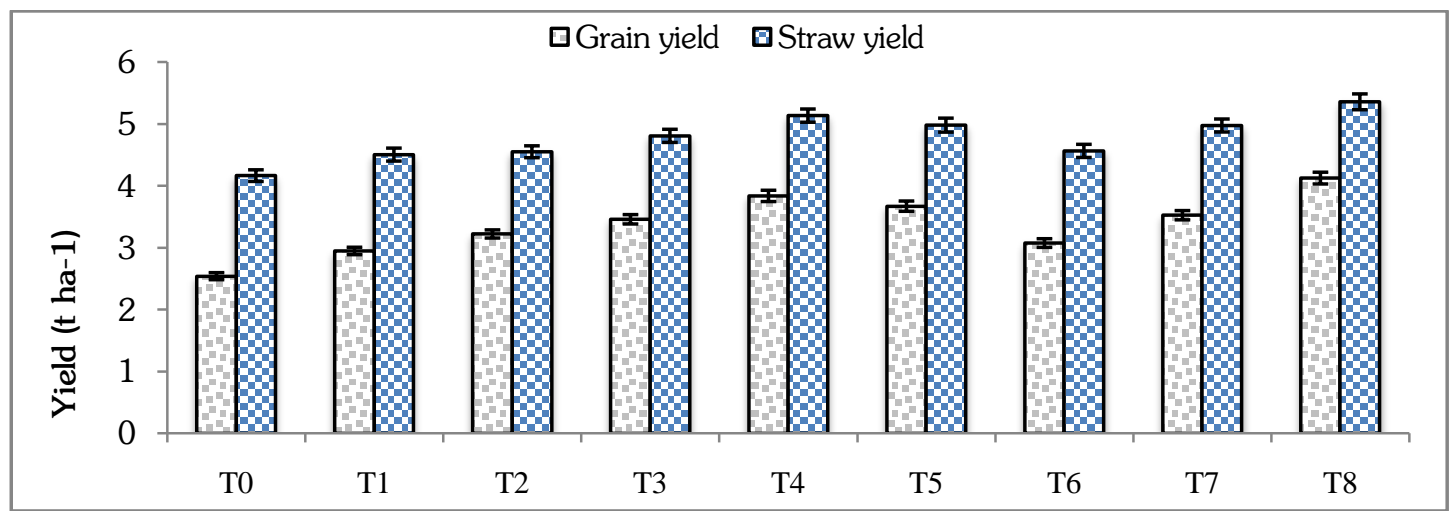

Fig. 2. Effect of organic and probiotic soil amendments on grain yield and straw yield of wheat in 2017-2018. 


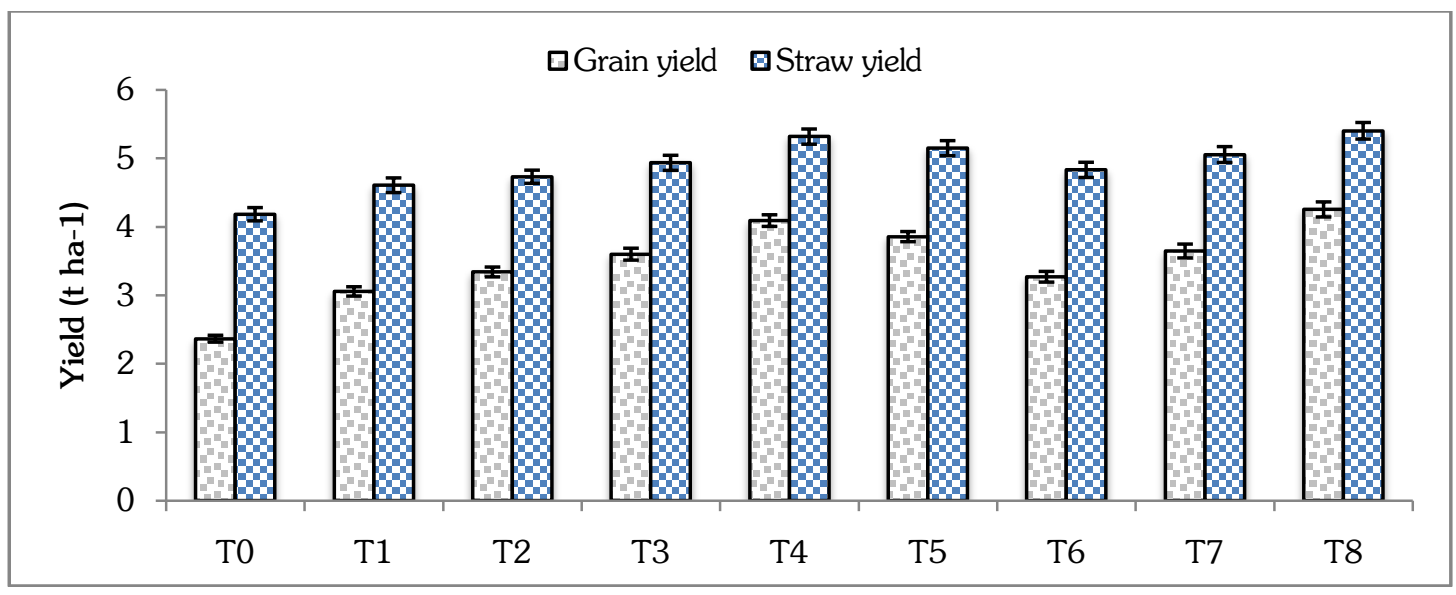

Fig. 3. Effect of organic and probiotic soil amendments on grain yield and straw yield of wheat in 2018-2019.

\section{Harvest index}

Treatment $\mathrm{T}_{8}$ showed a maximum harvest index (44.05\%) followed by $\mathrm{T}_{4}(43.48 \%)$ and the lowest one (36.13\%) derived from $\mathrm{T}_{0}$ during 2018-2019 (Figure 4). The boosting harvest index might be the continued supply of nutrients and higher dry matter distribution towards grain formation provided by the poultry manure treatment combination. This result was in agreement with the investigation of Kabesh et al. (2009) and Khan et al. (2018).

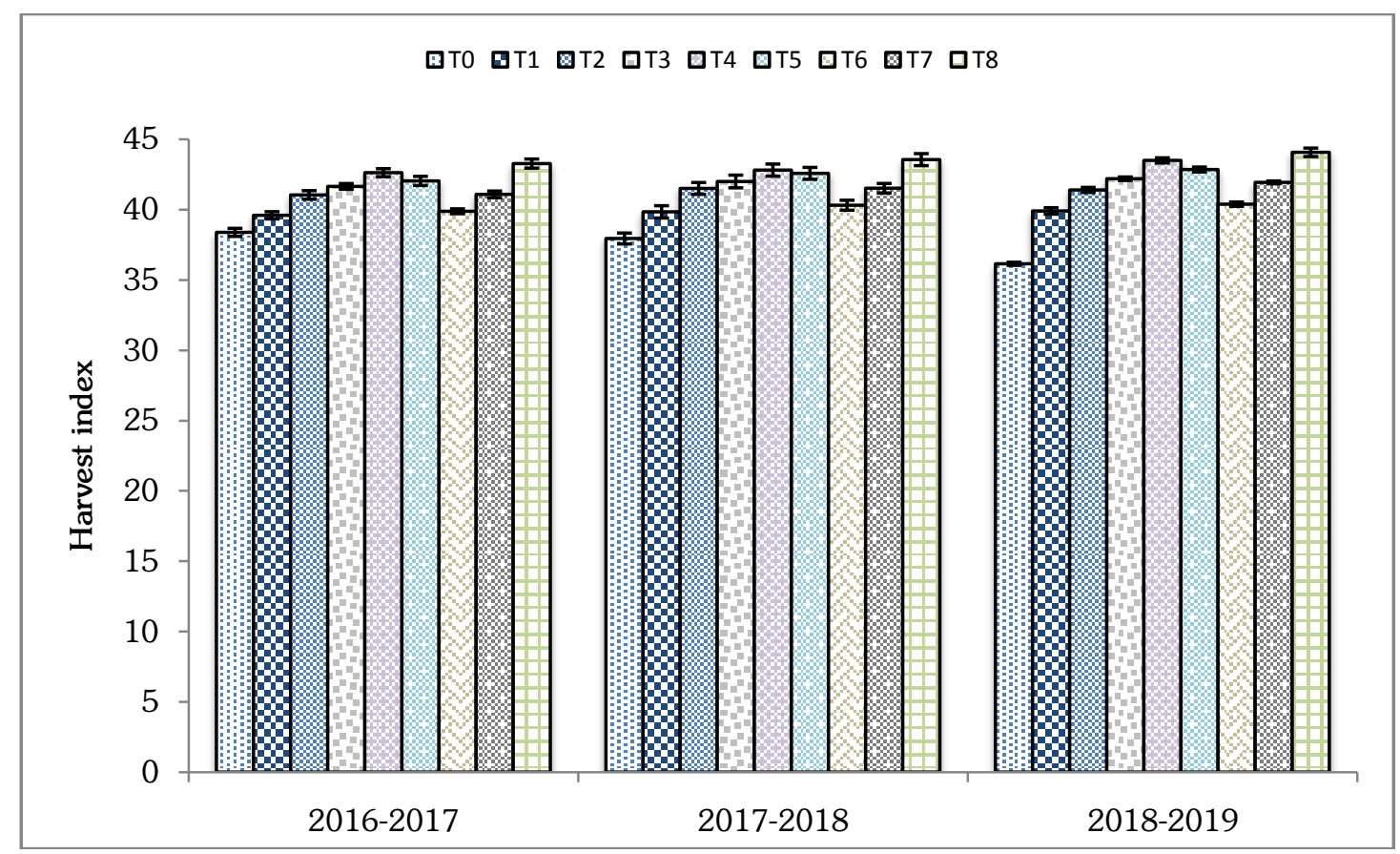

Fig. 4. Effect of organic and probiotic soil amendments on harvest index of wheat in 20162017, 2017-2018 and 2018-2019. 


\section{Effect of soil amendments on seed quality}

After every harvesting, seeds were collected separately from each treatment and preserved. Major seed quality characters viz. germination, vigor index and total soluble protein content were determined. The introduction of designed soil amendments not only developed the growth and yield of wheat but also enriched parameters of seed quality.

\section{Germination (\%)}

Soil amendments influenced the germination of seed. The treatment $\mathrm{T}_{4}$ significantly enhanced $(92.08 \%)$ the germination followed by $\mathrm{T}_{5}(88.89 \%)$ and $\mathrm{T}_{0}$ had a lower effect $(74.78 \%)$ on germination in 2018-2019 (Table 3). A similar trend was also observed in the previous two cropping season. This might be due to an adequate supply of nutrients which helps to build up carbohydrate-like seed elements thus producing vigorous seeds. The outcome of the investigation was the conformity of previous results of Gowda et al. (2010) and Aslam et al. (2011).

Table 3. Effect of organic and probiotic soil amendments on seed quality of wheat

\begin{tabular}{|c|c|c|c|c|c|c|c|c|c|}
\hline \multirow{2}{*}{ Treatments } & \multicolumn{3}{|c|}{ Germination (\%) } & \multicolumn{3}{|c|}{ Vigor index } & \multicolumn{3}{|c|}{$\begin{array}{l}\text { Soluble protein content(mg } \\
\left.\qquad \mathrm{g}^{-1} \mathrm{FW}\right)\end{array}$} \\
\hline & $\begin{array}{l}2016- \\
2017\end{array}$ & $\begin{array}{l}2017- \\
2018 \\
\end{array}$ & $\begin{array}{l}2018- \\
2019 \\
\end{array}$ & $\begin{array}{l}2016- \\
2017 \\
\end{array}$ & $\begin{array}{l}2017- \\
2018\end{array}$ & $\begin{array}{l}2018- \\
2019 \\
\end{array}$ & $\begin{array}{l}2016- \\
2017 \\
\end{array}$ & $\begin{array}{c}2017- \\
2018 \\
\end{array}$ & $\begin{array}{c}2018- \\
2019 \\
\end{array}$ \\
\hline $\mathrm{T}_{0}$ & $\begin{array}{l}70.50 h \\
\pm 0.92\end{array}$ & $\begin{array}{l}72.53 \mathrm{~g} \\
\pm 0.93\end{array}$ & $\begin{array}{l}74.78 \mathrm{~g} \\
\pm 0.85\end{array}$ & $\begin{array}{l}24.70 \mathrm{~h} \\
\pm 0.42\end{array}$ & $\begin{array}{l}27.49 f \\
\pm 0.40\end{array}$ & $\begin{array}{l}28.84 \mathrm{f} \\
\pm 0.27\end{array}$ & $\begin{array}{l}77.31 e \\
\pm 0.28\end{array}$ & $\begin{array}{l}77.47 e \\
\pm 0.58\end{array}$ & $\begin{array}{l}79.10 e \\
\pm 0.42\end{array}$ \\
\hline $\mathrm{T}_{1}$ & $\begin{array}{l}75.31 \mathrm{~g} \\
\pm 1.08\end{array}$ & $\begin{array}{l}77.54 \mathrm{f} \\
\pm 1.02\end{array}$ & $\begin{array}{l}79.67 f \\
\pm 1.18\end{array}$ & $\begin{array}{l}28.20 \mathrm{~g} \\
\pm 0.55\end{array}$ & $\begin{array}{l}32.52 e \\
\pm 0.54\end{array}$ & $\begin{array}{l}34.39 e \\
\pm 0.36\end{array}$ & $\begin{array}{l}78.20 e \\
\pm 0.45\end{array}$ & $\begin{array}{l}79.68 \mathrm{~d} \\
\pm 1.05\end{array}$ & $\begin{array}{l}79.58 e \\
\pm 0.52\end{array}$ \\
\hline $\mathrm{T}_{2}$ & $\begin{array}{l}78.14 f \\
\pm 0.82\end{array}$ & $\begin{array}{l}80.88 \mathrm{de} \\
\pm 0.98\end{array}$ & $\begin{array}{l}83.67 d \\
\pm 0.96\end{array}$ & $\begin{array}{l}31.69 e \\
\pm 0.85\end{array}$ & $\begin{array}{l}34.42 \mathrm{~d} \\
\pm 0.96\end{array}$ & $\begin{array}{l}35.93 d \\
\pm 0.38\end{array}$ & $\begin{array}{l}80.25 \mathrm{~d} \\
\pm 0.46\end{array}$ & $\begin{array}{l}80.97 \mathrm{~cd} \\
\pm 0.73\end{array}$ & $\begin{array}{l}82.98 \mathrm{~d} \\
\pm 0.39\end{array}$ \\
\hline $\mathrm{T}_{3}$ & $\begin{array}{l}81.08 \mathrm{~d} \\
\pm 0.85\end{array}$ & $\begin{array}{c}83.86 b c \\
\pm 0.92\end{array}$ & $\begin{array}{l}86.17 c \\
\pm 0.94\end{array}$ & $\begin{array}{l}32.60 \mathrm{~d} \\
\pm 0.43\end{array}$ & $\begin{array}{l}36.84 \mathrm{~b} \\
\pm 0.49\end{array}$ & $\begin{array}{l}38.36 b \\
\pm 0.34\end{array}$ & $\begin{array}{l}80.20 \mathrm{~d} \\
\pm 1.02\end{array}$ & $\begin{array}{l}81.98 c \\
\pm 1.44\end{array}$ & $\begin{array}{l}82.24 \mathrm{~d} \\
\pm 1.28\end{array}$ \\
\hline $\mathrm{T}_{4}$ & $\begin{array}{l}88.27 a \\
\pm 1.05\end{array}$ & $\begin{array}{l}89.93 a \\
\pm 1.01\end{array}$ & $\begin{array}{l}92.08 \mathrm{a} \\
\pm 1.24\end{array}$ & $\begin{array}{l}35.99 a \\
\pm 0.73\end{array}$ & $\begin{array}{l}39.48 \mathrm{a} \\
\pm 0.84\end{array}$ & $\begin{array}{l}41.54 a \\
\pm 0.58\end{array}$ & $\begin{array}{l}88.17 b \\
\pm 1.89\end{array}$ & $\begin{array}{l}93.30 \mathrm{a} \\
\pm 0.90\end{array}$ & $\begin{array}{l}92.56 b \\
\pm 0.70\end{array}$ \\
\hline $\mathrm{T}_{5}$ & $\begin{array}{l}84.75 b \\
\pm 1.06\end{array}$ & $\begin{array}{l}86.52 b \\
\pm 1.15\end{array}$ & $\begin{array}{l}88.89 \mathrm{~b} \\
\pm 0.84\end{array}$ & $\begin{array}{l}34.57 b \\
\pm 0.52\end{array}$ & $\begin{array}{l}37.30 \mathrm{~b} \\
\pm 0.58\end{array}$ & $\begin{array}{l}39.26 b \\
\pm 0.46\end{array}$ & $\begin{array}{l}87.59 b \\
\pm 0.59\end{array}$ & $\begin{array}{l}88.30 \mathrm{~b} \\
\pm 0.66\end{array}$ & $\begin{array}{l}91.03 c \\
\pm 0.99\end{array}$ \\
\hline $\mathrm{T}_{6}$ & $\begin{array}{l}78.76 e \\
\pm 0.89\end{array}$ & $\begin{array}{c}\text { 78.40ef } \\
\pm 1.46\end{array}$ & $\begin{array}{l}81.83 e \\
\pm 1.42\end{array}$ & $\begin{array}{l}30.21 \mathrm{f} \\
\pm 0.46\end{array}$ & $\begin{array}{l}34.68 \mathrm{~d} \\
\pm 0.33\end{array}$ & $\begin{array}{c}35.37 \mathrm{de} \\
\pm 0.33\end{array}$ & $\begin{array}{l}81.51 \mathrm{c} \\
\pm 0.72\end{array}$ & $\begin{array}{l}81.31 \mathrm{c} \\
\pm 0.63\end{array}$ & $\begin{array}{l}82.62 \mathrm{~d} \\
\pm 0.70\end{array}$ \\
\hline $\mathrm{T}_{7}$ & $\begin{array}{l}83.59 c \\
\pm 0.93\end{array}$ & $\begin{array}{c}83.33 \mathrm{~cd} \\
\pm 1.05\end{array}$ & $\begin{array}{l}86.28 c \\
\pm 0.96\end{array}$ & $\begin{array}{l}33.56 c \\
\pm 0.43\end{array}$ & $\begin{array}{l}35.78 c \\
\pm 0.38\end{array}$ & $\begin{array}{l}36.98 c \\
\pm 0.42\end{array}$ & $\begin{array}{l}87.88 \mathrm{~b} \\
\pm 0.58\end{array}$ & $\begin{array}{l}89.17 b \\
\pm 0.83\end{array}$ & $\begin{array}{c}91.99 b c \\
\pm 0.67\end{array}$ \\
\hline $\mathrm{T}_{8}$ & $\begin{array}{l}81.37 d \\
\pm 0.86\end{array}$ & $\begin{array}{l}84.57 b c \\
\pm 1.05\end{array}$ & $\begin{array}{r}86.76 c \\
\pm 01.16\end{array}$ & $\begin{array}{l}32.46 \mathrm{~d} \\
\pm 0.50\end{array}$ & $\begin{array}{l}34.03 \mathrm{~d} \\
\pm 0.34\end{array}$ & $\begin{array}{l}37.27 c \\
\pm 0.48\end{array}$ & $\begin{array}{l}93.32 \mathrm{a} \\
\pm 1.08\end{array}$ & $\begin{array}{l}93.80 \mathrm{a} \\
\pm 1.20\end{array}$ & $\begin{array}{l}96.43 a \\
\pm 1.14\end{array}$ \\
\hline LS & *** & *** & $* *$ & *** & $* *$ & $* *$ & $* *$ & **** & $* *$ \\
\hline CV (\%) & 0.82 & 3.34 & 1.79 & 0.97 & 3.16 & 2.91 & 1.58 & 1.61 & 1.42 \\
\hline
\end{tabular}

In the column, mean values bearing similar letter(s) or without letter are similar and those having dissimilar letters are differed significantly as per Duncan's Multiple Range Test. $T_{0}=$ control, $T_{1}=$ rice straw + vermicompost + green manure, $T_{2}=$ cow dung + vermicompost + green manure, $T_{3}=$ compost + vermicompost + green manure, $\mathrm{T}_{4}=$ poultry manure + vermicompost + green manure, $\mathrm{T}_{5}=T$. harzianum + vermicompost + green manure, $\mathrm{T}_{6}=$ mungbean residues + vermicompost + green manure, $\mathrm{T}_{7}=T$. viride + vermicompost + green manure, $\mathrm{T}_{8}=$ chemical fertilizer, $\mathrm{LS}=$ Level of significance, ${ }^{* *}=1 \%$ level of significance, $C V=$ Coefficient of variation 


\section{Vigor index}

The vigor index is an imperative quality of seed that indicates the potentiality of constant emergence of seedlings (Pradeep, 2018). After three years of experimentation, considering the comparable trend of vigor index from respective treatments, poultry manure (41.54) and Trichoderma harzianum (39.26) in comparison with control treatment (28.84). The speed of germination i.e. vigor index might be significantly different for providing the substantial macro and micro nutrient in the grain filling stage and carbohydrate stored in seed enhances the quality. This result was in agreement with the reflection of Farhad et al. (2011).

\section{Total soluble protein content}

Among the major cereals, wheat is the prominent source of vegetal protein (13\%). The results in general $\mathrm{T}_{8}$ produced higher protein $\left(96.43 \mathrm{mg} \mathrm{g}^{-1} \mathrm{FW}\right)$ followed by $\mathrm{T}_{4}\left(92.56 \mathrm{mg} \mathrm{g}^{-1} \mathrm{FW}\right)$ and the least at $\mathrm{T}_{0}\left(79.10 \mathrm{mg} \mathrm{g}^{-1} \mathrm{FW}\right)$. The increase of protein content might be due to the availability of high NPK that satisfy the plant requirement and accumulated $\mathrm{N}$ in leaf mobilized to seed components which leads the protein production. This result was in corroborates with the findings of Abedi et al. (2010) and Rasul et al. (2015). Considering the fact, the poultry manure combination treatment $\left(\mathrm{T}_{4}\right)$ was an environmentally friendly alternative to chemical fertilizer $\left(\mathrm{T}_{8}\right)$ for increasing soil fertility and crop productivity.

\section{Conclusion}

There is a potentiality for sustainable improvement of wheat growth, yield and seed quality with organic soil amendment through probiotic and organic manure. In that term, the inclusion of green manure, vermicompost and poultry manure might be a worthy alternative. Organic manures particularly poultry manure was a healthier source for improvement of growth, yield parameters and seed quality of wheat as compared to other manures. So, the poultry manures could be suggested for soil amendment and increase wheat production in the studied region.

\section{References}

Abedi, T., A. Alemzadeh and S.A. Kazemeini. 2010. Effect of organic and inorganic fertilizers on grain yield and protein banding pattern of wheat. Aust. J. Crop Sci. 4:384-389.

Ahmed, B.E.A.M., A.A. Ishag, M.K. Hassan and M.O. Ahmed. 2017. Response of two wheat cultivars (Triticum aestivum L.) to amended nitrogen fertilizer on yield and grain quality in Halfa Elgadidah area. MOJ. Biol. Med. 1(5): 133-136.

Ahmed, S.I., M.K. Abbasi and G. Rasool. 2002. Integrated plant nutrition system (IPNS) in wheat under rain fed conditions of Rawalakot Azad Jammu and Kashmir [Pakistan]. Pak. J. Soil Sci. 21(1\&2): 79-86.

Alam, M.M., M.R. Karim and J.K. Ladha. 2013. Integrating best management practices for rice with farmers' crop management techniques: A potential option for minimizing rice yield gap. Field Crops Res. 144: 62-68.

Aslam, M., M.A. Khan, I.U. Awan, E.A. Khan, A.A. Khan and G. Jilani. 2011. Effect of single and combined use of various organic amendments on wheat grown over green manured soil: growth and yield attributes. Pak. J. Nutr. 7: 640-646.

Auti, A.K., S.C. Wadile and V.S. Pawar. 1999. Yield, quality and nutrient removal of wheat (Triticum aestivum L.) as influenced by levels and sources of fertilizer. Indian J. Agron.44: 119-120.

BARI (Bangladesh Agricultural Research Institute). 2014. Krishi Projukti Hatboi (Handbook on Agrotechnology), $6^{\text {th }}$ edition, Gazipur, Bangladesh. 
BARI (Bangladesh Agricultural Research Institute).2011. Krishi Projukti Hatboi (Handbook on Agrotechnology), $5^{\text {th }}$ edition, Gazipur, Bangladesh.

BBS (Bangladesh Bureau of Statistics). 2008. Statistical Yearbook of Bangladesh. Statistics and Informatics Div., Ministry of Planning. Govt. People's Repub., Bangladesh, Dhaka.

BBS (Bangladesh Bureau of Statistics).2017.Statistical Yearbook of Bangladesh. Statistics and Informatics Div., Ministry of Planning. Govt. People's Repub., Bangladesh, Dhaka.

Farhad. W, M.F. Saleem, M.A. Cheema, H.Z. Khan and H.M. Hammad. 2011. Influence of poultry manure on the yield and quality of spring maize. Crop Environ. 2: 6-10.

Garg, S. and G.S. Bahla. 2008. Phosphorus availability to maize as influenced by organic manures and fertilizer P associated phosphatase activity in soils. Bioresour. Technol. 99: 5773-5777.

Gomez. K.A. and A.A. Gomez.1984. Statistical Procedures for Agricultural Research (2nd edition). International Rice Research Institute. John Wiley and Sons, a Wiley-Inter Science Publication.

Gowda, C., P.N.K. Biradar, B.N. Patil, J.S. Awakknavar and R. Hunje. 2010. Effect of organic manures on growth, seed yield and seed quality of wheat. Karnataka J. Agric. Sci. 21: 366368.

Guy, C., D. Haskell, L. Neven, P. Klein and C. Smelser. 1992. Hydration-state-responsive proteins link cold and drought stress in spinach. Planta. 188: 265-270.

Hajieghrari, B. and M. Mohammadi. 2016. Growth-promoting activity of indigenous Trichoderma isolates on wheat seed germination, seedling growth and yield. Aust. J. Crop Sci.10: 13391347.

Hue, N.V. and J.A. Silva. 2000. Organic Soil Amendments for Sustainable Agriculture: Organic Sources of Nitrogen, Phosphorus and Potassium: Plant nutrient management in Hawaii's soils, approaches for tropical and subtropical agriculture. College of Tropical Agriculture and Human Resources, University of Hawaii, Manoa. 133-144.

ISTA (International Seed Testing Association). 1985. International rules for seed testing. Seed Sci. Technol. 13: 299-513.

Kabesh, M.O., M.F. El-Kramany, G.A. Sary, H.M. El-Naggar and S.H. Gehan. 2009. Effects of sowing methods and some bio-organic fertilization treatments on yield and yield components of wheat. Res. J. Agric. Biol. Sci. 5: 97-102.

Khan, T.U., M.T. Jan, A. Khan, G. Ahmad, M. Ishaq, K. Afridi, A. Murad, M.A. Qureshi, I. Ahmad and M. Seed. 2018. Integrated management of fertilizer nitrogen and poultry manure enhances wheat production. Pak. J. Agric. Res. 31: 207-215.

Kumar, S. and T. Abraham. 2018. Productivity potential of wheat under certified organic production system. Int. J. Curr. Microbiol. Appl. Sci. 7: 281-288.

Maguire, J.D. 1962. Speed of germination-aid in selection and evaluation for seedling emergence and vigor. Crop Sci. 2: 176-177.

Metho, L.A., P.S. Hammes and E.A. Beyers. 1998. The effect of soil fertility on the contribution of main stem, tillers and kernel position to grain yield and grain protein content of wheat. S. Afr. J. Plant Soil 15: 53-60.

Mukhtiar, A., A. Waqar, M.K. Khalil, M. Tariq, S. Muhammad, A. Hussain and A. Kamal. 2018. Evaluating the potential organic manure for improving wheat yield and quality under agroclimatic conditions of Pakistan. Adv. Crop Sci. Tech. 6: 1-4.

Nguyen, M.L., R.J. Haynes and K.M. Goh. 1995. Nutrient budgets and status in three pairs of conventional and alternative and mixed cropping farms in Canterbury, New Zealand. Agric. Ecosyst. Environ. 52: 149-162. 
Peltonen-sainio, P., A. Kangas, Y. Salo and L. Jauhiainen. 2007. Grain numbers dominate grain weight in temperate cereal yield determination: evidence based on 30 years of multi-location trials. Field Crops Res. 100: 179-188.

Pradeep, P. 2018. Seed quality parameters (Germination percentage and seedling vigor index) of rabi sorghum seeds influenced by rice weevil infestation. MOJ. Toxicol. 4: 391-396.

Pradhan, S.B. 1992. Status of fertilizer use in developing countries of Asia and the Pacific Region In: Proc. Reg. FADINAP Sem. Chiang Mai, Thailand: 37-47.

Rasul, G.A.M., S.T. Ahmed and M.Q. Ahmed. 2015. Influence of different organic fertilizers on growth and yield of wheat. American-Eurasian J. Agric. Environ. Sci. 15: 1123-1126.

Sabaghina, N., M. Janmohammadi and A.E. Segherloo. 2014. Evaluation of some agromorphological traits diversity in Iranian bread wheat genotypes. Annales UMCS, Biologia 69: 79-92.

Shah, S.A., S.M. Shah, W. Mohammad, M. Shafi, H. Nawaz, S. Shehzadi and M. Amir. 2010. Effect of integrated use of organic and inorganic nitrogen sources on wheat yield. Sarhad J. Agric. 26: 559-563.

Shewry, P.R., N.G. Halford, P.S. Belton and A.S. Tatham. 2002. The structure and properties of gluten: An elastic protein from wheat grain. Philosophical Transactions of the Royal Society of London Series B: Biol. Sci. 357: 133-142.

Siavoshi, M.,A. Nasiri and S.L. Laware. 2011. Effect of organic fertilizer on growth and yield components in rice (Oryza sativa L.). J. Agric. Sci. 3:217.224.

Singh, A., M.S. Darhvankar and G. Singh. 2018. Impact of organic and inorganic amendments on yield and growth of wheat (Triticum aestivum L.). Int. J. Curr. Microbiol. Appl. Sci. 7: 789794.

Thiry, D.E., R.G. Sears, J.P. Shroyer and G.M. Paulsen. 2002. Relationship between tillering and grain yield of Kansas wheat varieties. Kansas State University Agricultural Experimental Station and Cooperative Extension Service: 1-5.

Yadvinder-Singh, S. Bijay, R.K. Gupta, J. Ladha, J.S. Bains and J. Singh. 2008. Evaluation of press mud cake as a source of nitrogen and phosphorus for the rice-wheat cropping system in the Indo-Gangetic plains of India. Biol. Fertil. Soils. 45: 289-296.

Zeidan, M.S. and M.F.E. Kramany. 2001. Effect of organic manure and slow-release N Fertilizer on the productivity of wheat (Triticum aestivum L.) in sandy soil. Acta Agronomica Hungarica. 49: 379-385.

Zhang, H.Q., X.Y. Yu, B.N. Zhai, Z.Y. Jin and Z.H. Wang. 2016. Effect of manure under different nitrogen application rates on winter wheat production and soil fertility in dry land. IOP Conf. Ser.: Earth Environ. Sci. 39(1):1-10. 